\title{
How do branched detergents stabilize GPCRs in micelles?
}

Sangbae Lee, ${ }^{1}$ Soumadwip Ghosh, ${ }^{1}$ Suvamay Jana, ${ }^{1}$ Nathan Robertson, ${ }^{2}$ Christopher G.

Tate, ${ }^{3}$ Nagarajan Vaidehi ${ }^{*}, 1$

'Department of Computational and Quantitative Medicine, Beckman Research Institute of the City of Hope, 1500 E. Duarte Road, Duarte, California 91010, USA

${ }^{2}$ Heptares Therapeutics Ltd, BioPark, Broadwater Road, Welwyn Garden City, AL7 3AX, UK

3MRC Laboratory of Molecular Biology, Cambridge Biomedical Campus, Francis Crick Avenue, Cambridge CB2 oQH, UK.

\section{Supporting Information}




\section{SI Methods}

Calculation of RMSD, RMSF and $\alpha$-helicity: The root mean squared deviation (RMSD) of the backbone atoms of the receptor was calculated using the gmx rms module. RMSD gives a measure of how far the structures deviate from the chosen reference structure (starting structure) during the simulations and is used to determine the convergence of each simulation (Figure 3A and S3A). The root mean square fluctuation (RMSF) of the backbone atoms of the receptor was calculated using gmx rmsf module of GROMACS. RMSF is a measure of the flexibility of each residue in the protein with respect to the average structure calculated form the MD simulation trajectories. To render the extent of flexibility on the receptor structure as a heat map, we converted RMSF to thermal B-factor using B-factor $=\left(8 \mathrm{p} \pi^{2} / 3\right)(\mathrm{RMSF})^{2}$. The average structure calculated from the combined trajectories ( 5 trajectories) for each system was used as the reference structure for the RMSF calculations. We calculated the $\alpha$-helicity of every residue in the transmembrane region of the GPCR, using helicity.tcl VMD script which uses STRIDE to identify if a residue is in helical conformation. ${ }^{[1]}$ The average helicity was calculated for the transmembrane residues of receptors (Figure 3 and Figure S3).

Conformational Clustering Method: We performed conformational clustering using RMSD cutoff of $1.5 \AA$ from the aggregated trajectories from the five simulations for any given system using the script gmx_cluster. We used gromos method for conformational clustering. ${ }^{[2]}$ The representative structure of the most populated cluster was calculated as the frame that has the smallest RMSD to the center of this cluster of conformations. The most representative structure has been used in the figures.

Calculation of enthalpic energies: The energy components shown in all figures were calculated using GROMACS. Non-bond interaction energy included the Coulombic and Lennard-Jones potentials for receptor energy, receptor-detergent interaction energy, and receptor-water interaction energy. Water molecules within $5 \AA$ from the receptor were considered for the calculation of interaction energy between receptor and water. We compared only the non-bond energies of the antagonist bound $\mathrm{A}_{2 \mathrm{~A}} \mathrm{R}$ and wild type $\beta_{2} \mathrm{AR}$ in LMNG, DMNG, OGNG, and DDM detergent micelles.

Calculation of eccentricity of the receptor-detergent micelles: The eccentricity of the ellipse of the four receptor-detergent micelles of $\mathrm{tA}_{2 \mathrm{~A}} \mathrm{R}$ (Figure 2B) and $\beta_{2} \mathrm{AR}$ (Figure S2C) is defined as the ratio of the long axes to the short axes. The reported results of eccentricity in this study were calculated using gmx gyrate module of GROMACS package with the specific option -moi.

Calculation of radial distribution function $(R D F)$ : For analyses of how the detergent molecules organize around the receptor $\mathrm{tA}_{2 \mathrm{~A}} \mathrm{R}$ (Figure 4) and $\beta_{2} \mathrm{AR}$ (Figure S4), we calculated RDF for detergent molecules from the center of mass of receptor using gmx $r d f$ module of the GROMACS package.

Calculation of spatial distribution function (SDF): The spatial distribution function for four detergents for $\mathrm{t}_{2 \mathrm{~A}} \mathrm{R}$ and $\beta_{2} \mathrm{AR}$ (Figure $4 \mathrm{~B}$ and $\mathrm{S} 4 \mathrm{~B}$, respectively) was calculated using the program tool gmx spatial in the GROMACS package. 
Calculation of the number of inter-helical hydrogen bond and van der Waals interactions: We first identified all the receptor residues that make interhelical hydrogen bond or van der Waals contact in more than $50 \%$ from the last $100 \mathrm{~ns}$ of MD snapshots. The hydrogen bond interactions were calculated beteen heteroatoms (N, O) with a distance cutoff of $3.5 \AA$ and angle cutoff of $120^{\circ}$ using gmx hbond Gromacs tool. The van der Waals (vdW) contacts were calculated with a distance cutoff of $4.0 \AA$ between two sidechain carbon atoms using VMD tool ContactFreq.tcl (Figure 1 and Figure $\mathrm{S} 1$ ).

Calculation of Volumetric density of water: We generated the volumetric density maps of tail carbon atoms of detergent within $4 \AA$ of each receptor (Figure 2 and Figure S2). The densitytype volume was contoured using VolMap tool of VMD software. All the above properties were calculated for last 500ns of the MD simulation trajectories at which time all the properties had equilibrated and fluctuations minimized. 
Table S1. Thermostability as apparent $\mathrm{T}_{\mathrm{m}}\left({ }^{\circ} \mathrm{C}\right)$ of GPCRs under various conditions.

\begin{tabular}{llll}
\hline & $\mathrm{T}_{\mathrm{m}}\left({ }^{0} \mathrm{C}\right)$ & System & Refs. \\
\hline LMNG & $44.2 \pm 0.2$ & Inactive $\mathrm{A}_{2 \mathrm{~A}} \mathrm{R}$ Mutant & Current work \\
DMNG & $33.9 \pm 0.2$ & & \\
OGNG & $24.2 \pm 0.6$ & & \\
\hline LMNG & $68.4 \pm 0.09$ & Inactive $\beta_{2} \mathrm{AR}$ wild type & Chae, P. S., et al., Nat. Method 2010 \\
DDM & $63.5 \pm 0.16$ & & \\
\hline LMNG & $66 \pm 1$ & Inactive $\mathrm{A}_{2 \mathrm{~A}} \mathrm{R}$ Mutant & Ashok, Y., et al., Protein Eng. Des. Sel. 2015 \\
DDM & $62 \pm 1$ & & \\
\hline LMNG & $51 \pm 2$ & Inactive $\mathrm{A}_{2 \mathrm{~A}} \mathrm{R}$ wild type & Ashok, Y., et al., Protein Eng. Des. Sel. 2015 \\
DDM & $40 \pm 2$ & & \\
\hline
\end{tabular}


Table S2. (Related to Methods section). List of all systems for which MD simulations were performed used in the current study.

\begin{tabular}{lccccc}
\hline \multicolumn{1}{c}{ System } & PDB & Ligand & $\begin{array}{c}\text { No. } \\
\text { Detergent }\end{array}$ & $\begin{array}{c}\text { Equilibration } \\
\text { Time }\end{array}$ & $\begin{array}{c}\text { Production } \\
\text { Time }\end{array}$ \\
\hline Inactive $\mathrm{A}_{2 \mathrm{~A}} \mathrm{R}$ & $3 \mathrm{PWH}$ & ZM241385 & $96 \mathrm{LMNG}$ & $18 \mathrm{~ns}$ & $1.25 \mu \mathrm{s}$ \\
Mutant $\left(\mathrm{tA}_{2 \mathrm{~A}} \mathrm{R}\right)$ & $3 \mathrm{PWH}$ & & $96 \mathrm{DMNG}$ & $18 \mathrm{~ns}$ & $1.25 \mu \mathrm{s}$ \\
& $3 \mathrm{PWH}$ & & $96 \mathrm{OGNG}$ & $18 \mathrm{~ns}$ & $1.25 \mu \mathrm{s}$ \\
& $3 \mathrm{PWH}$ & & $192 \mathrm{DDM}$ & $18 \mathrm{~ns}$ & $1.25 \mu \mathrm{s}$ \\
\hline Inactive $\beta_{2} \mathrm{AR}$ & $2 \mathrm{RH} 1$ & Carazolol & $96 \mathrm{LMNG}$ & $18 \mathrm{~ns}$ & $1.25 \mu \mathrm{s}$ \\
wild type & $2 \mathrm{RH} 1$ & & $96 \mathrm{DMNG}$ & $18 \mathrm{~ns}$ & $1.25 \mu \mathrm{s}$ \\
& $2 \mathrm{RH} 1$ & & $96 \mathrm{OGNG}$ & $18 \mathrm{~ns}$ & $1.25 \mu \mathrm{s}$ \\
& $2 \mathrm{RH} 1$ & & $192 \mathrm{DDM}$ & $18 \mathrm{~ns}$ & $1.25 \mu \mathrm{s}$ \\
\hline Inactive $\mathrm{A}_{2 \mathrm{~A}} \mathrm{R}$ & $3 \mathrm{PWH}$ & $\mathrm{ZM} 241385$ & $96 \mathrm{LMNG}$ & $18 \mathrm{~ns}$ & $1.0 \mu \mathrm{s}$ \\
Wild type $\left(\mathrm{WT}-\mathrm{A}_{2 \mathrm{~A}} \mathrm{R}\right)$ & $3 \mathrm{PWH}$ & & $192 \mathrm{DDM}$ & $18 \mathrm{~ns}$ & $1.0 \mu \mathrm{s}$ \\
\hline
\end{tabular}



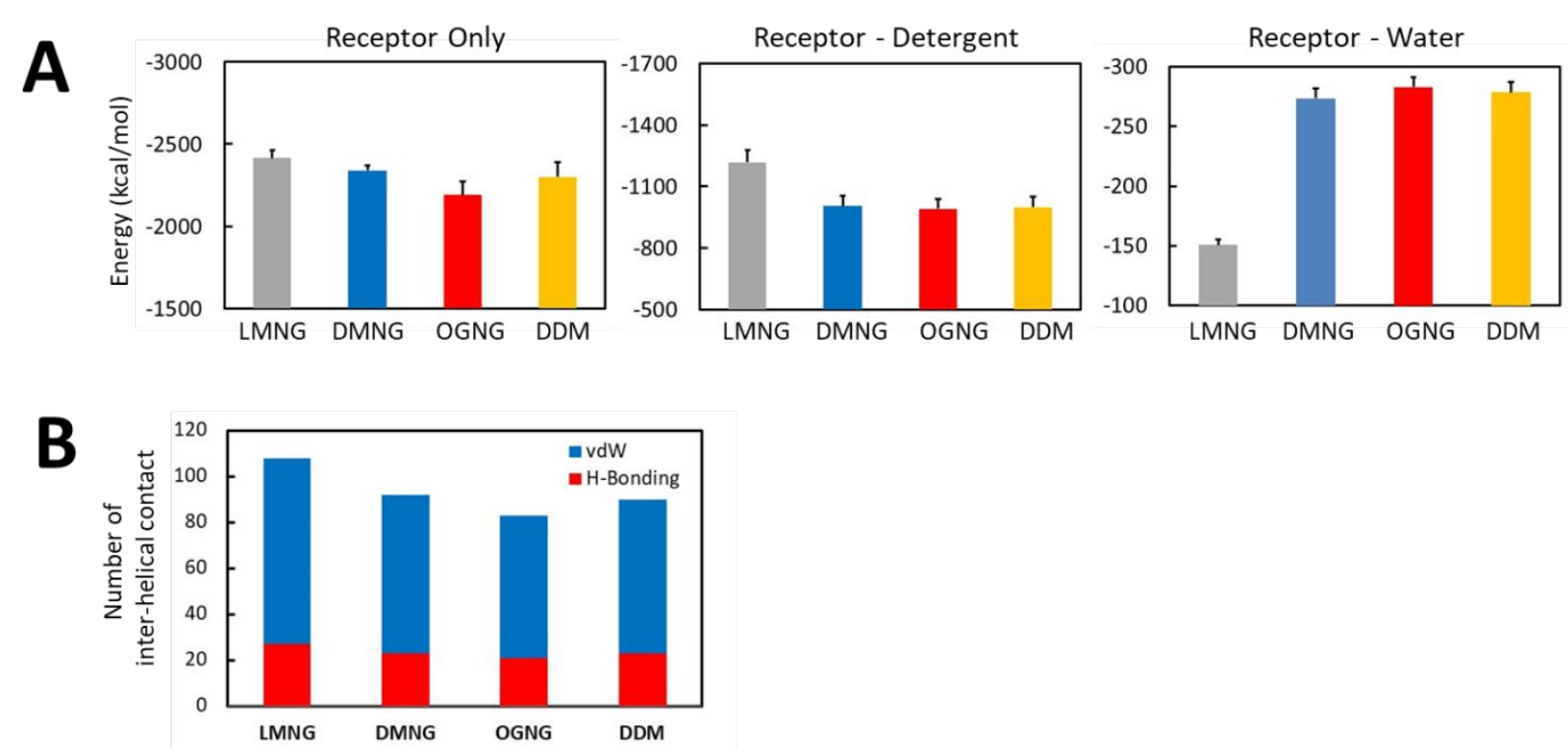

Figure S1. (Related to Figure 1). A. Calculated nonbond energies of the transmembrane region of the receptor, receptor-detergent interaction energy, and receptor-water interaction energy in $\beta_{2} \mathrm{AR}$. B. The number of inter-helical interactions in each receptor-detergent micelle. In red is the number of sustained (for more than $50 \%$ of the MD snapshots) inter-helical hydrogen bonds and in blue is the number of interhelical van der Waals interactions for $\beta_{2} \mathrm{AR}$. 
A

\begin{tabular}{l|cccc}
\hline & LMNG & DMNG & OGNG & DDM \\
\hline $\mathrm{A}_{2 \mathrm{~A}} \mathrm{R}$ & $0.72( \pm 0.09)$ & $0.86( \pm 0.05)$ & $0.90( \pm 0.06)$ & $0.70( \pm 0.05)$ \\
& & & & $0.80( \pm 0.07)$ \\
$\beta_{2} \mathrm{AR}$ & $0.76( \pm 0.07)$ & $0.91( \pm 0.06)$ & $0.95( \pm 0.03)$ & $0.76( \pm 0.06)$ \\
\hline
\end{tabular}

C

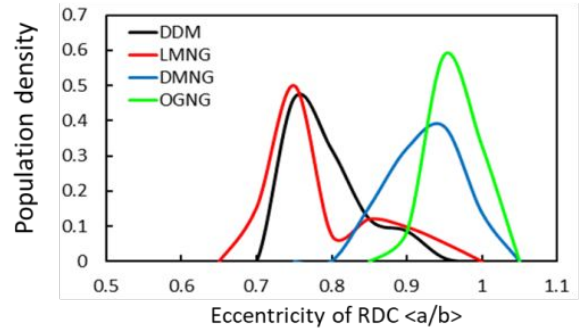

D

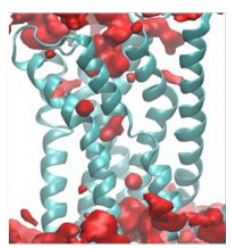

LMNG

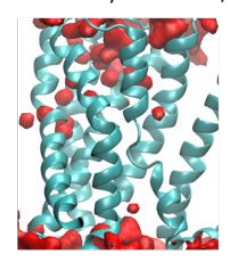

DMNG

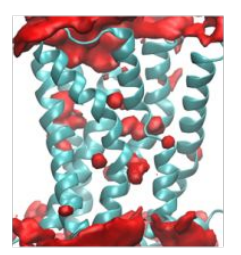

OGNG

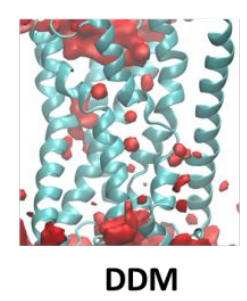

B
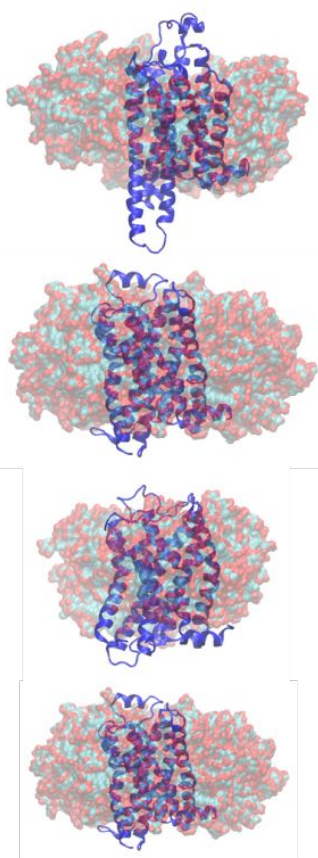

DMNG

OGNG

LMNG

DDM

Figure S2. (Related to Figure 2). A. Calculated eccentricities as average values for four receptor-detergent complexes in the $\mathrm{tA}_{2 \mathrm{~A}} \mathrm{R}$ and $\beta_{2} \mathrm{AR}$. B. Molecular geometry of three RDC (receptor-detergent complex) in $\beta_{2} A R$. C. Distribution of the eccentricity of RDC (receptor-detergent complex) in four different detergents for $\beta_{2} \mathrm{AR}$. D. Water density display within 3.5 angstrom of $\beta_{2} \mathrm{AR}$ receptor. 
A
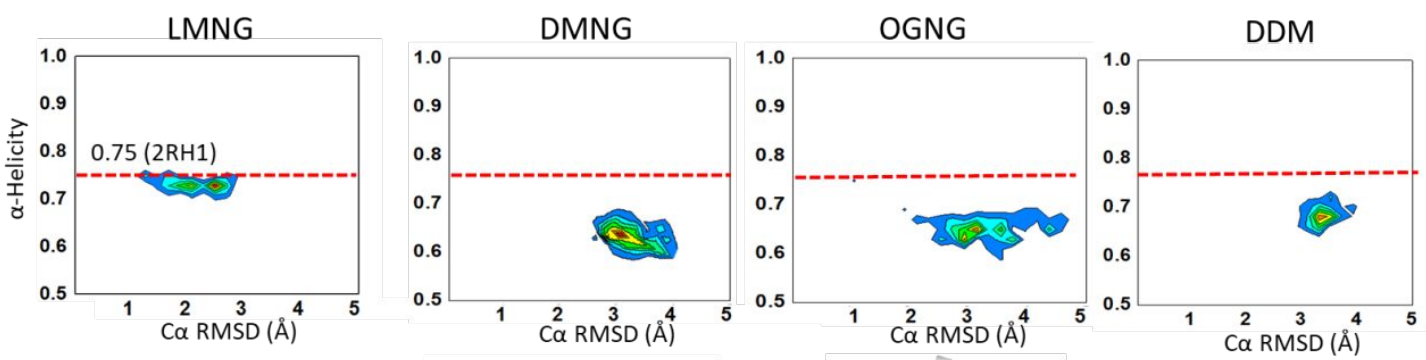

B
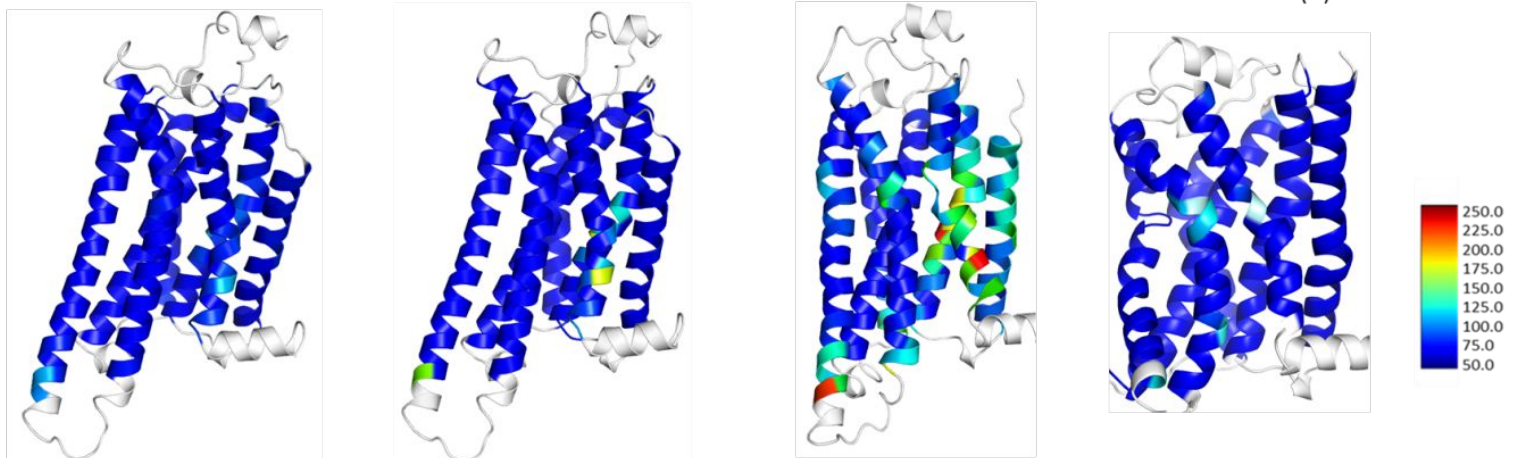

Figure S3. (Related to Figure 3). Conformational heterogeneity of inactive state of $\beta_{2} A R$ in LMNG, DMNG, OGNG, and DDM detergents. A. conformations from MD simulations of $\beta_{2} \mathrm{AR}$ projected in $\alpha-$ helicity and RMSD space. The RMSD was calculated with respect to the respective crystal structures. The red-dotted lines in the figure are the $\alpha$-helical content of the crystal structure of $\beta_{2} \mathrm{AR}$. B. The RMSF of residues in the TM regions shown in heat-maps of LMNG, DMNG, OGNG, and DDM detergent complexes from RMSF in $\beta_{2} \mathrm{AR}$. The loop regions and helix8 shown in white for which RMSF are not shown for clarity. 
A
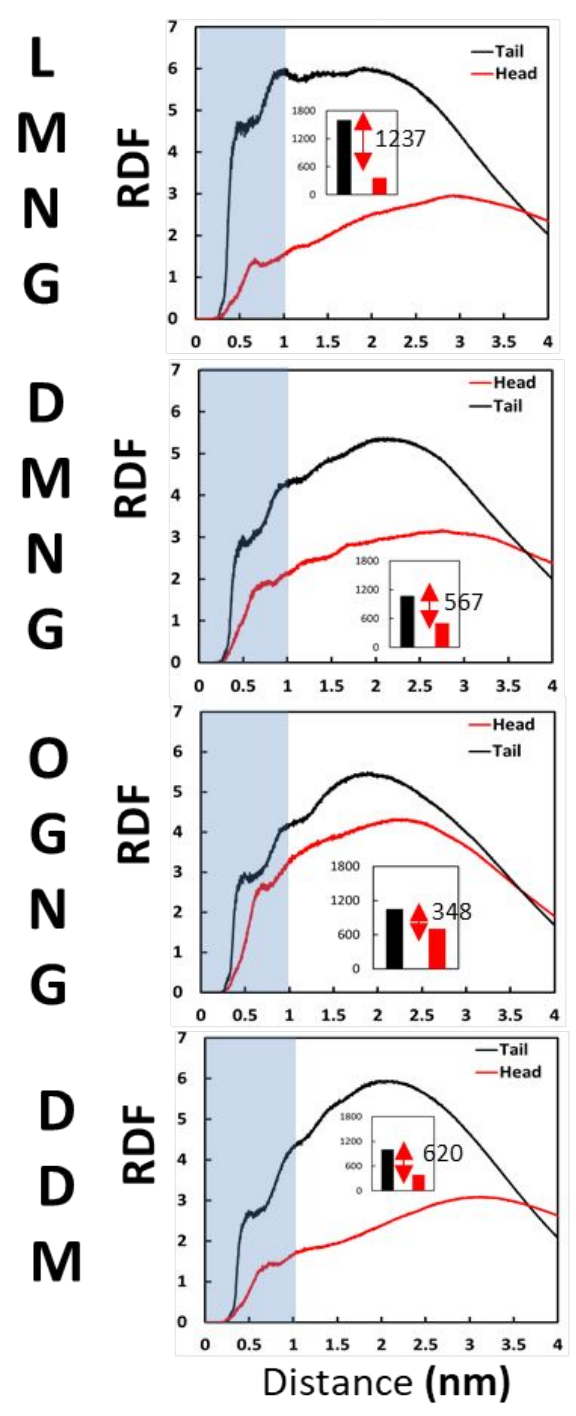

B
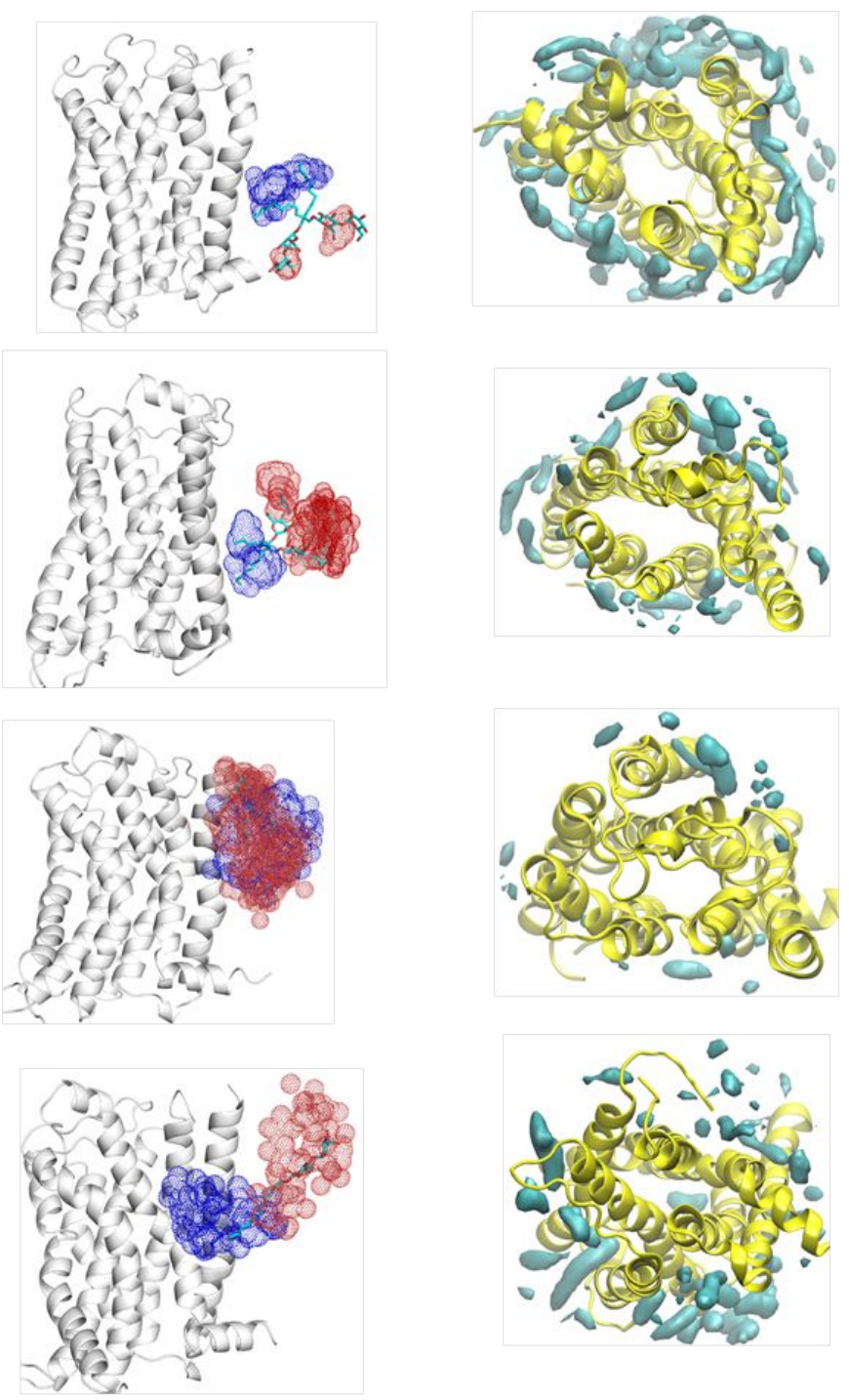

Figure S4. (Related to Figure 4). Characteristics of the detergent micelle around inactive state of $\beta_{2} \mathrm{AR}$. A. RDF plot for the density for either the head-group (red) or tail-group (black) of the detergents as a function of distance from $\beta_{2} \mathrm{AR}$ receptor in $\mathrm{LMNG}$, DMNG, OGNG, and DDM systems. Inset figure shows the difference between the shaded areas for tail to head-group distributions within $1.0 \mathrm{~nm}(10 \AA)$. B. Spatial distribution functions of the detergent molecules for LMNG, DMNG, OGNG, and DDM detergent near the $\beta_{2} \mathrm{AR}$ shown for the most populated cluster. C. Volumetric density of tail carbon within $4 \AA$ of each residue in the $\beta_{2} \mathrm{AR}$ receptors. 

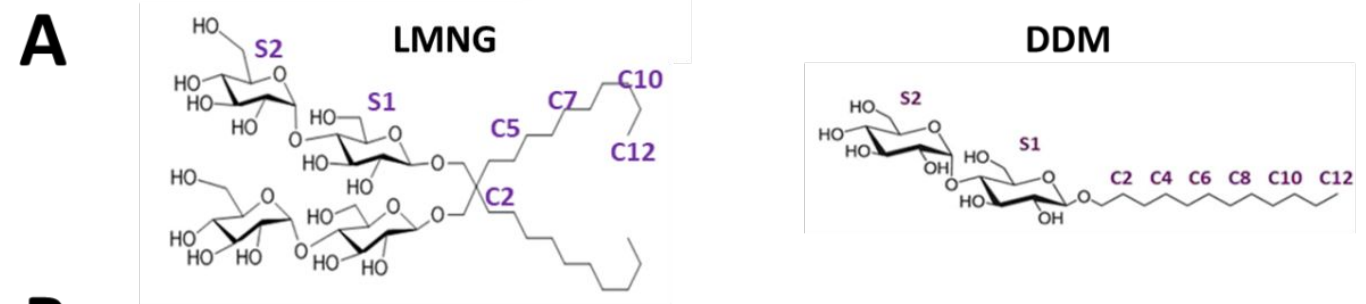

B
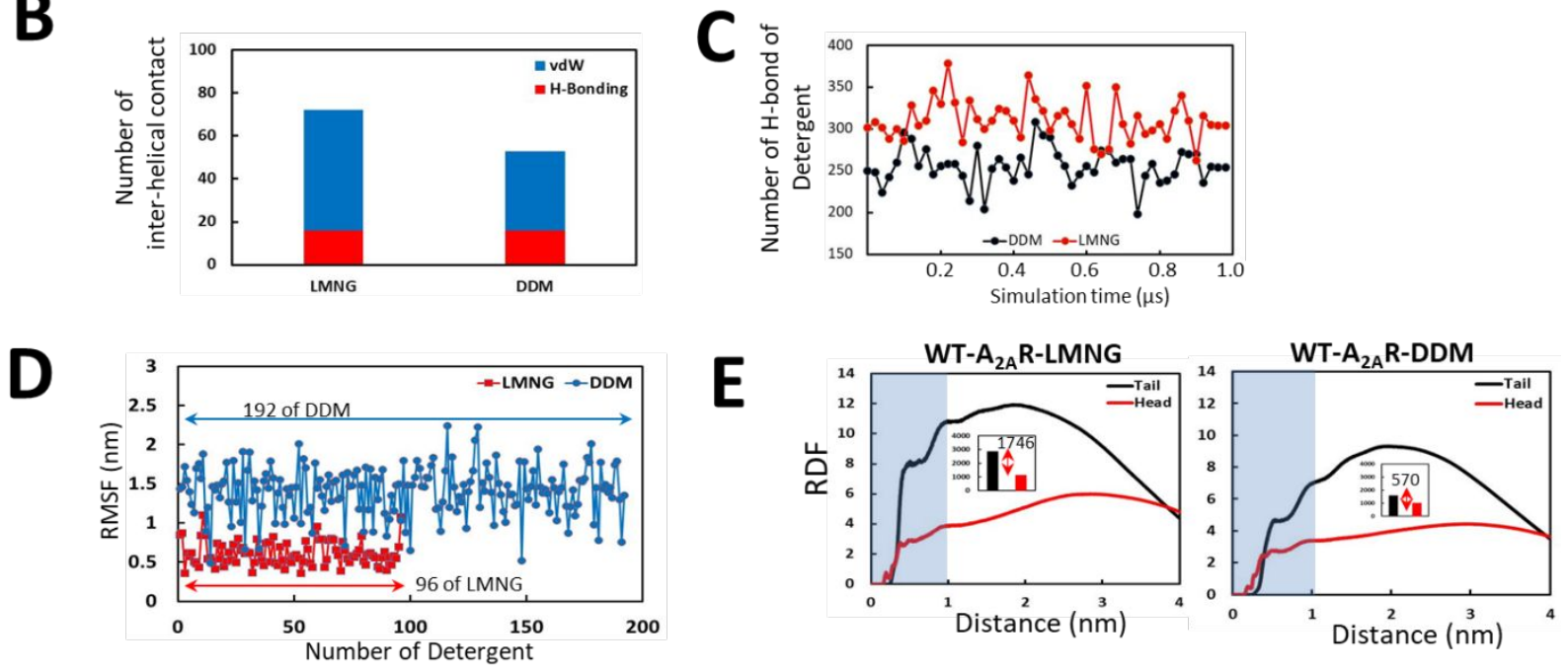

Figure S5. (Related to Figure 5). Effect of branched amphiphilic detergent LMNG compared to single alkyl chain DDM detergent on inactive state of wild type $A_{2 A} R$ (WT- $A_{2 A} R$ ). A. 2D Structures of LMNG and DDM detergent we used. B. The number of sustained inter-helical interactions of LMNG- WT- $\mathrm{A}_{2 \mathrm{~A}} \mathrm{R}$ and DDM-WT- $\mathrm{A}_{2 \mathrm{~A}} \mathrm{R}$ complexes that are present in more than $50 \%$ of conformations in MD trajectories. $\mathbf{C}$. The number of inter-molecular H-bond interaction between the head group of the LMNG molecules in the micelle, and between the head groups of the DDM detergents as a function of MD simulation time. D. Flexibility RMSF, of each detergent molecule (192 for DDM and 96 of LMNG) in the wild type of $A_{2 A} R$ with LMNG (red) and DDM (blue) detergent complex. E. RDF plots for density for head- and tail-group of LMNG (left) and DDM (right) detergent in the wild type simulations. Inset figure shows the difference between the shaded areas for tail to head-group distributions within $1.0 \mathrm{~nm}(10 \AA)$. 


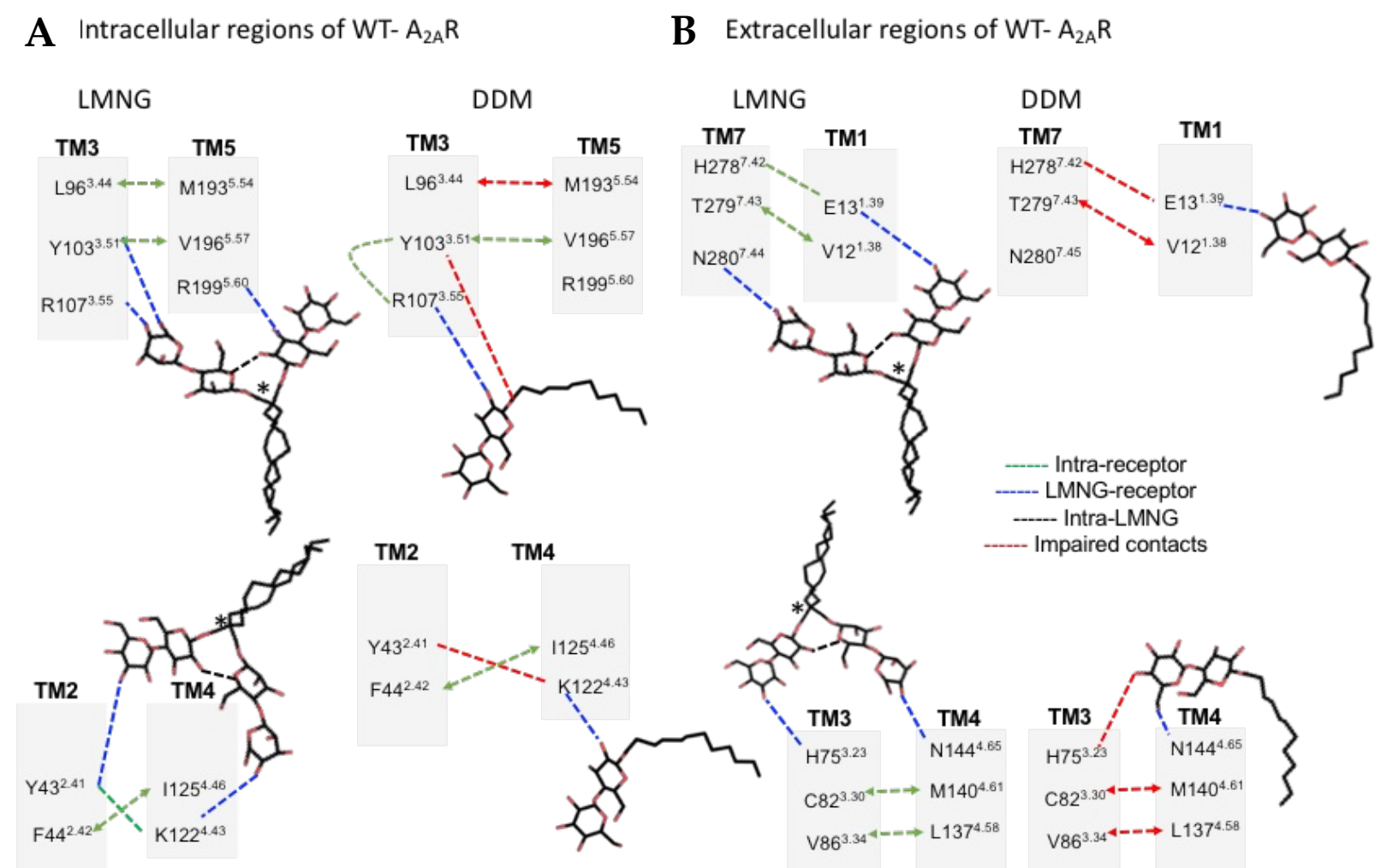

Figure S6 (Related to Figure 6): Schematic figures of LMNG and DDM forming hydrogen bonds between TM helices. A. The bifurcated hydrogen bonds formed between the edge of TM helices and intracellular loops are shown for LMNG on the left. DDM does not form such bifurcated hydrogen bonds as illustrated on the right panel. B. The hydrogen bonds formed by LMNG and DDM in the extracellular TM and loop regions. The quaternary carbon atom of LMNG is denoted by an asterisk in each scheme. 


\section{REFERENCES}

(1) Frishman, D.; Argos, P. Knowledge Based Protein Secondary Structure Assignment. Proteins 1995, 23(4), 566-579

(2) Daura, X.; Gademann, K.; Jaun, B.; SeebAch, D.; van Gunsteren, W. F. V.; Mark, A. E. Peptide Folding: when Simulation Meets Experiments. Angew. Chem. Int. Ed. 1999, 38, 236-240. 